\title{
Origem Anômala das Artérias Coronárias
}

\author{
Flavio Henrique Amaral Pires Veras ${ }^{1}$, Edgar Guimarães Victor ${ }^{1}$,
} Lurildo Cleano Ribeiro Saraiva ${ }^{1}$, Marly Maria Uellendahl Lopes ${ }^{1}$

\section{RESUMO}

A origem anômala das artérias coronárias é uma doença potencialmente letal, sobretudo em lactentes e atletas jovens. O entendimento da fisiopatologia da doença permitiu a diferenciação entre as diferentes apresentações clínicas. Apesar de ser causa de isquemia miocárdica, os exames indutores de isquemia podem ser normais. A cineangiocoronariografia foi durante muito tempo considerada o exame ideal para o diagnóstico da doença, mas os exames de imagem cardiovascular não-invasivos têm mostrado uma melhor definição da origem e do trajeto das artérias coronárias. O tratamento cirúrgico deve ser indicado em pacientes sintomáticos, principalmente em crianças com origem anômala da artéria coronária esquerda da artéria pulmonar. O manejo ideal para os pacientes assintomáticos permanece indefinido.

DESCRITORES: Anomalias dos vasos coronários. Morte súbita. Diagnóstico por imagem. Técnicas de diagnóstico cardiovascular.

\section{SUMMARY}

\section{Anomalous Origin of Coronary Arteries}

The anomalous origin of the coronary arteries is a potentially lethal pathology, especially in neonates and young athletes. The understanding of the pathophysiological pattern of the disease has allowed the recognition of the different forms of clinical presentation. Despite being a cause of myocardial ischemia, functional tests can be normal. Coronary angiography used to be the gold standard for the diagnosis of this pathology, but non-invasive cardiovascular imaging tests have shown better definition of the origin and course of coronary arteries. Surgical treatment should be indicated for symptomatic patients, mainly in children with the anomalous origin of the left coronary artery from the pulmonary artery. The ideal management of asymptomatic patients remains undefined.

DESCRIPTORS: Coronary vessel anomalies. Death, sudden. Diagnostic imaging. Diagnostic techniques, cardiovascular.

indivíduo pode ser assintomático até antes do evento letal $\left.\right|^{1,12}$. Os exames complementares, sobretudo os de imagem cardiovascular, são ferramentas importantes para o diagnóstico.

Na origem anômala da artéria coronária esquerda da artéria pulmonar (OACEP), o tratamento cirúrgico tem excelentes resultados ${ }^{10,13}$. A terapêutica da origem anômala das artérias coronárias da aorta (OACEA) é objeto de constante discussão.

\section{CLASSIFICAÇÃO E DEFINIÇÃO}

A primeira classificação para anomalias das artérias coronárias foi proposta por Ogden ${ }^{14}$, em 1969. Esta classificação subdividia as anomalias coronarianas em maiores, menores e secundárias, com bases anatômicas, mas não propunha correlação clínica ${ }^{15}$.

Dodge-Khatami et al. ${ }^{15}$ subdividiram as anomalias coronarianas em sete categorias, de acordo com critérios de complexidade clínica. Estas seriam: artérias coronárias originando-se da artéria pulmonar, artérias coronárias com origem anômala na aorta, atresia congênita do tronco coronariano esquerdo, fístulas arteriovenosas coronarianas, artérias coronarianas

\footnotetext{
${ }^{1}$ Hospital das Clínicas da Universidade Federal de Pernambuco Recife, PE.

Correspondência: Flavio Henrique A. P. Veras. Serviço de Cardiologia do Hospital das Clínicas da UFPE. Av. Prof. Moraes Rego, SN Cidade Universitária - Recife, PE - CEP: 50670-240 Tel.: (81) 2126-3714 - E-mail: fhveras@bol.com.br Recebido em: 6/5/2007 • Aceito em: 29/7/2007
} 
formando pontes miocárdicas, aneurismas de artérias coronarianas e estenose coronariana.

Para Angelini et al. ${ }^{8}$, uma anomalia coronariana deve ser definida como qualquer padrão coronariano com uma característica (número de óstios, trajeto proximal, leito distal...) "raramente" encontrado na população geral. Sendo favorável às seguintes definições: normal, qualquer característica morfológica observada em mais de $1 \%$ de uma população não selecionada; variante da normalidade, uma característica morfológica relativamente incomum prevalente em mais de 1\% da mesma população; e anomalia, uma característica morfológica vista em menos de $1 \%$ dessa população.

A anatomia coronariana normal, universalmente aceita, é assim definida ${ }^{16,17}$ :

- o tronco coronariano origina-se do seio coronariano esquerdo e dividi-se na artéria descendente anterior e na artéria circunflexa;

- a artéria descendente anterior segue posteriormente ao tronco pulmonar no sulco interventricular anterior;

- a artéria circunflexa segue no sulco atrioventricular posterior;

- a artéria coronária direita origina-se do seio coronariano direito e segue pelo sulco atrioventricular anterior.

Qualquer alteração neste padrão deve ser considerada como uma anatomia coronariana anormal ${ }^{16,17}$. A artéria coronária circunflexa originando-se do seio coronariano direito ou da artéria coronária direita, com um trajeto retroaórtico, é a anomalia congênita da artéria coronária mais comum ${ }^{18,19}$.

\section{FISIOPATOLOGIA}

Anomalias coronarianas têm sido implicadas como causa de dor torácica, morte súbita, insuficiência cardíaca, síncope, dispnéia, fibrilação ventricular e infarto do miocárdio ${ }^{8}$.

Na OACEP, observa-se que durante a vida intrauterina não acontece nenhuma alteração funcional, entretanto, após o nascimento, na medida em que cai a resistência arteriolar e decresce a pressão da artéria pulmonar, diminui a saturação oxi-hemoglobínica do sangue venoso, fecham-se o canal arterial e o forame oval, diminuindo progressivamente a perfusão sangüínea do miocárdio irrigado pela artéria anômala, levando a alterações isquêmicas ${ }^{10}$.

A OACEP, geralmente, trata-se de um defeito isolado, mas pode associar-se, em 5\% dos casos, a outros defeitos cardíacos, como comunicação interventricular, comunicação interatrial, coarctação da aorta, tetralogia de Fallot e outras anomalias ${ }^{13}$. Em portadores de tetralogia de Fallot, a incidência de anomalia coronariana pode variar de $3 \%$ a $36 \%^{20,21}$.
A atresia congênita do tronco coronariano esquerdo (TCE) é uma anomalia extremamente rara e geralmente de evolução benigna ${ }^{22}$. Em alguns casos, pode levar a isquemia miocárdica e morte súbita ${ }^{22}$. Os portadores desta anomalia sobrevivem graças ao desenvolvimento de circulação colateral para a artéria descendente anterior ${ }^{22}$. A circulação colateral pode ser formada por ramos da artéria do cone, também conhecida como Círculo de Vieussens; ou anastomoses entre os ramos ventriculares anteriores da artéria coronária direita e a artéria descendente anterior; ou anastomoses das ramificações terminais da artéria coronária direita com ramos terminais da artéria descendente anterior ${ }^{22,23}$.

A explicação para isquemia miocárdica na atresia do TCE, na ausência de aterosclerose ou vasoespasmo, não é clara e a justificativa mais lógica é o fato de que uma artéria coronariana única é incapaz de suprir, adequadamente, a demanda sangüínea miocárdica ${ }^{22}$.

A origem anômala da artéria coronária esquerda do seio coronariano direito pode relacionar-se a morte súbita em 59\% dos casos, precedida por atividade física em $81 \%$ dos eventos ${ }^{8}$. Esta anomalia pode apresentar quatro trajetos: anteriormente a artéria pulmonar; posteriormente a aorta; intra-septal entre a aorta e a artéria pulmonar; e interarterial entre a aorta e a pulmonar (Figura 1$)^{24}$. Todos os subtipos desta anomalia têm sido associados à morte súbita, mas a variação interarterial, que é o padrão mais comum, tem uma relação mais forte com seqüelas catastróficas ${ }^{1,8,24-26}$.

Potenciais mecanismos têm sido levantados para explicar a presença de isquemia miocárdica e morte súbita em pacientes com OACEA: a formação de um ângulo agudo e contorcido na origem da coronária anômala da aorta; um estreitamento no orifício coronariano secundário à anatomia anômala; compressão da artéria coronária anômala durante o seu trajeto entre a aorta e o tronco pulmonar no exercício; e espasmo da artéria anômala, possivelmente como resultado de lesão endotelial $\left.\right|^{8,27,28}$.

De acordo com as evidências atuais, os segmentos coronarianos com trajeto anômalo não são mais susceptíveis a doença obstrutiva aterosclerótica do que os segmentos normais num mesmo indivíduo ${ }^{8,9}$.

\section{QUADRO CLÍNICO}

A OACEP apresenta duas faixas de apresentação: uma forma infantil e uma forma adulta. A forma infantil é caracterizada pela pobreza de circulação colateral intercoronariana, podendo resultar em infarto do miocárdio, insuficiência cardíaca ou morte súbita ${ }^{11}$. Nesta forma de apresentação, a sintomatologia ocorre nos primeiros meses de vida, com o aparecimento de choro constante, palidez intensa, parada súbita da sucção, perda de peso e sinais de insuficiência cardíaca, como taquipnéia, taquicardia, cansaço às mama- 


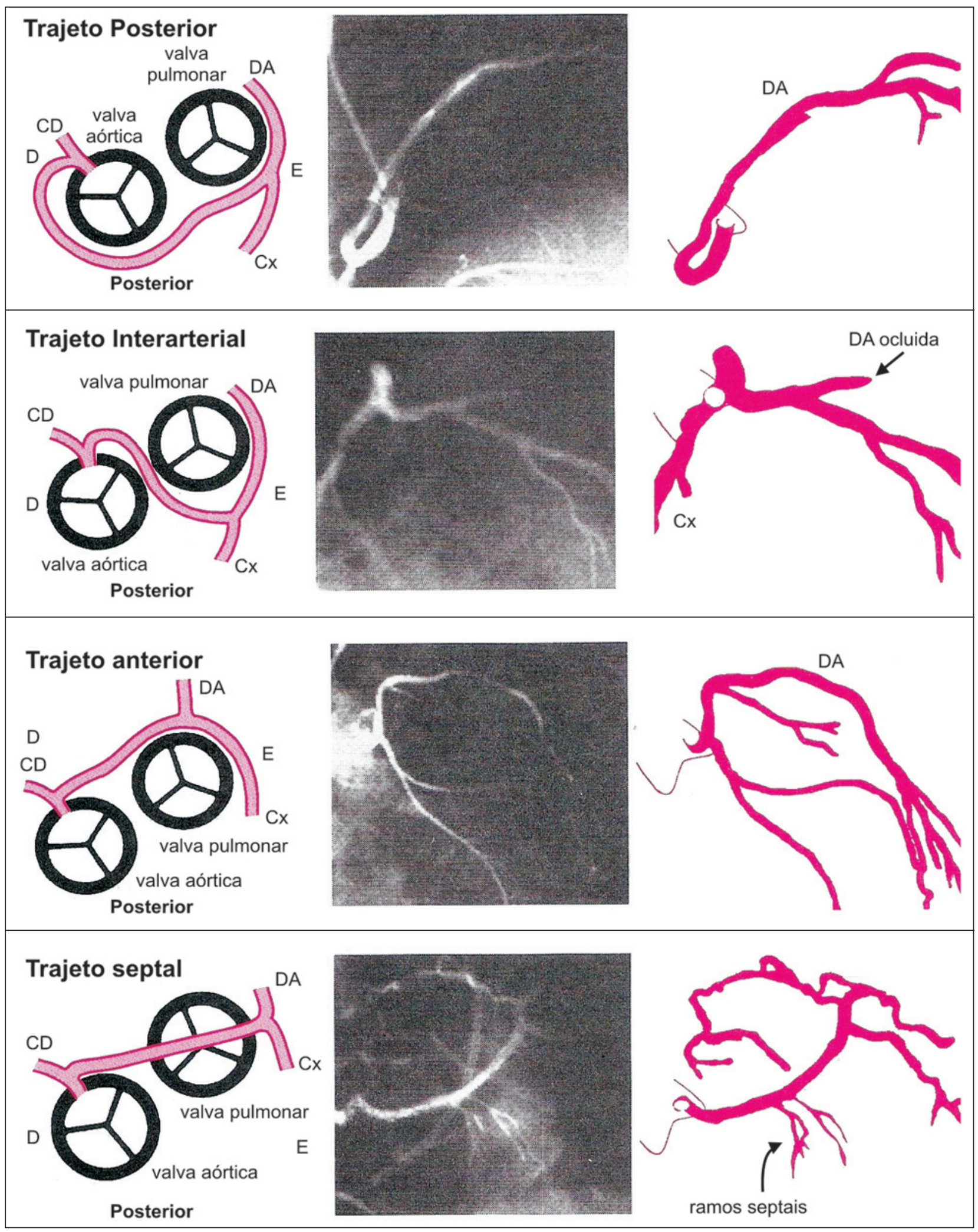

Figura 1 - Origem anômala da artéria coronária esquerda no seio de Valsalva direito. Cada painel tem uma representação de um corte craniocaudal, ao nível das válvulas semilunares, mostrando o trajeto da artéria coronária anômala. Os angiogramas em posição oblíqua anterior direita e as representações esquemáticas mostram exemplos dos quatro trajetos mais comuns de artéria coronária esquerda anômala originandose do seio de Valsalva: posterior (retroaórtico), interarterial, anterior e septal. CD: artéria coronária direita, DA: artéria descendente anterior: Cx: artéria circunflexa (adaptado de Popma et al. ${ }^{27}$ ). 
das, sudorese, hepatomegalia e cianose periférica. Na ausculta, pode-se observar o ritmo de galope, abafamento de bulhas, principalmente da primeira e, ocasionalmente, o aparecimento de sopro sistólico na área mitral, devido à insuficiência mitral secundária ao aumento da área cardíaca, por sua dilatação ou por disfunção do músculo papilar ${ }^{10,11}$. Estima-se em $90 \%$ a mortalidade no primeiro ano de vida, mesmo com o tratamento clínico ${ }^{10,11}$.

A forma adulta ou madura pode ocorrer na criança e, necessariamente, no adulto em cerca de 10-15\% dos casos. Caracteriza-se por uma riqueza de circulação colateral intercoronariana, de magnitude suficiente para permitir uma sobrevida até a vida adulta, relatando-se sobrevida até os 72 anos de idade ${ }^{11}$.

Algumas vezes não há manifestações clínicas apreciáveis e o diagnóstico de OACEP é suspeitado pela presença de sopros: contínuo, pela passagem de sangue através da fístula coronariopulmonar, ou sistólico, por disfunção da valva mitral ${ }^{10}$. Alguns pacientes evoluem para insuficiência cardíaca, outros poderão apresentar angina do peito $^{10,11}$.

Na OACEA, alguns sintomas, como síncope, dor torácica e palpitação, podem estar presentes ${ }^{12,28}$. Mas a maioria dos portadores é assintomática e a única expressão desta anomalia coronariana em atletas jovens pode ser a morte súbita ${ }^{12,28}$. Os estudos sugerem que estas anomalias coronarianas podem ser letais apenas durante ou imediatamente após a realização de atividade física extenuante, tipicamente em indivíduos jovens ${ }^{8}$ (Tabela 1).

\section{EXAMES COMPLEMENTARES}

\section{Eletrocardiograma (ECG) e teste ergométrico (TE)}

Um estudo demonstrou que a presença de onda "Q" com profundidade maior que $3 \mathrm{~mm}$, duração maior que $30 \mathrm{~ms}$ e um padrão "QR" em pelo menos uma das derivações $\mathrm{DI}, \mathrm{aVL}, \mathrm{V} 5-\mathrm{V} 7$, esteve presente em $100 \%$ dos eletrocardiogramas de pacientes com OACEP ${ }^{29}$. A ausência de onda " $\mathrm{Q}$ " na parede inferior também é um achado prevalente ( $82 \%$ dos casos) ${ }^{13,15,29}$. Menos freqüente é o desvio do eixo para esquerda $(64 \% \text { dos casos })^{13}$. Alterações eletrocardiográficas atípicas podem mascarar a doença quando associadas a lesões com hipofluxo pulmonar importante ${ }^{13}$.

Na OACEA, o ECG e o TE são quase sempre normais ${ }^{8,9,12,28}$. Apesar da OACEA ser causa de isquemia miocárdica, esta é episódica, manifestando-se sob condições clínicas extenuantes $8,9,12,28$.

\section{Radiografia de tórax}

Em geral, mostra-se alterada nos casos associados à insuficiência cardíaca. Na OACEP, crianças de pouca idade podem apresentar aumento da área cardíaca ${ }^{10}$. A circulação pulmonar é normal, entretanto, em presença de insuficiência cardíaca, quadro de congestão venosa pulmonar pode ser observado ${ }^{10}$.

\section{Ecocardiograma}

O ecocardiograma bidimensional com Doppler colorido é útil na avaliação morfofuncional do coração. Para o diagnóstico de origem anômala das artérias coronárias, os estudos mostram uma sensibilidade variável, com melhores resultados em crianças ${ }^{11,13,15,26}$.

O ecocardiograma transesofágico (ETE) é um exame com sensibilidade muito maior para detecção de origem anômala de artérias coronárias ${ }^{1,26}$. O ETE, sobretudo com o uso de uma sonda multiplanar, é capaz de delinear com precisão o trajeto proximal e o padrão de fluxo nas artérias coronárias anômalas ${ }^{1,26}$.

\section{Tomografia computadorizada de artérias coronárias (TCAC)}

Os estudos mostram uma rápida evolução deste método não-invasivo, na avaliação das artérias coronárias. Inicialmente, os estudos com a Electron Beam Computed Tomography (EBCT) demonstraram excelente acurácia na avaliação da origem e trajeto proximal das anomalias coronarianas ${ }^{19,25}$.

Com o advento da tomografia computadorizada com múltiplos detectores (16 e 64 aquisições), passouse a ter uma melhor avaliação dos segmentos médio e distal para detecção de doença arterial coronariana obstrutiva ${ }^{16}$. Utiliza radiação ionizante e faz-se necessário o uso de medicação para diminuir a freqüência cardíaca $^{25}$. O método necessita da administração de contraste venoso, potencialmente alergênico e nefrotóxico.

TABELA 1

Incidência de morte súbita relacionada a anomalias de artérias coronárias (adaptado de Angelini et al.8).

\begin{tabular}{lcc}
\hline Grupo (idade) & Número de mortes & Mortes relacionadas a anomalias coronarianas, \% \\
\hline Indivíduos fazendo exercício (8-66 a) & 550 & 11 \\
População geral (<40 a) & 162 & 0,6 \\
Atletas competitivos(idade média de 17 a) & 134 & 23 \\
Corredores (30-46 a) & 120 & 1,6 \\
Indivíduos fazendo exercício, Maryland - EUA & 62 & 0 \\
\hline
\end{tabular}


Dados da literatura mostram ser um método seguro, eficaz e com excelente resolução espacial ${ }^{16,24,25,30}$. Os estudos comparativos com a angiografia coronariana têm mostrado eficácia superior, sugerindo que a tomografia computadorizada com múltiplos detectores seja o exame ideal para o diagnóstico de anomalias coronarianas $^{16,19}$.

A Figura 2 ilustra uma TCAC onde se evidencia uma OACEA com um curso interarterial.

\section{Ressonância nuclear magnética (RNM) das artérias coronárias}

A angiografia coronária por RNM é um exame não-invasivo, com elevada acurácia para identificação anatômica da origem e trajeto proximal das artérias coronárias ${ }^{17,31-34}$. A livre escolha do plano de imagem, com uma visão tridimensional, é importante vantagem sobre as limitadas possibilidades de angulação da angiografia coronariana convencional ${ }^{31}$. Utiliza contraste não-nefrotóxico e não usa radiação ionizante ${ }^{16}$.

Tem as desvantagens do longo tempo de duração do exame (45-50 min.), a necessidade de períodos de apnéia e a limitação na análise dos segmentos coronarianos distais ${ }^{16,31-34}$. Bunce et al. ${ }^{34}$ desenvolveram uma técnica para detecção de anomalias coronarianas onde não há necessidade de apnéia, com menor duração do exame (26 min., em média).

É um exame que possibilita avaliação cardíaca morfofuncional, sendo de grande utilidade na presença de cardiopatias congênitas, por permitir uma perfeita avaliação espacial entre as artérias coronárias, os grandes vasos e as demais estruturas cardíacas ${ }^{19,31-34}$.
A Figura 3 ilustra uma RNM diagnosticando uma atresia do TCE.

\section{Cineangiocoronariografia}

É tradicionalmente considerado o exame ideal para o diagnóstico das anomalias coronarianas. É um exame invasivo, que utiliza contraste nefrotóxico e radiação ionizante.

A evolução da RNM e da TCAC, ambos com análise tridimensional, tem evidenciado falhas na CA para o diagnóstico de anomalias coronarianas ${ }^{19,31-33}$. A CA pode ser incapaz de diferenciar, no trajeto da coronária anômala, a anatomia interarterial, de alto risco, da anatomia septal, retroaórtica ou da parede anterior, assim como a identificação do seio coronariano e a morfologia do óstio coronariano na parede da aorta ${ }^{19,31-33}$.

A CA ainda é o melhor método para avaliação do leito distal coronariano e de lesões ateroscleróticas associadas $^{19,35,36}$.

\section{TRATAMENTO}

Uma vez diagnosticada, a OACEP deve ser cirurgicamente corrigida logo em seguida ao diagnóstico, para prevenção de complicações e seqüelas próprias da história natural da doença ${ }^{10,11,13,37-40}$.

A técnica cirúrgica de eleição é o reimplante com translocação da artéria coronária esquerda do tronco pulmonar para a aorta, o que é possível na maioria dos $\operatorname{casos}^{11,13,37-40}$. Na impossibilidade de aplicação desta técnica, recomenda-se a "tunelização" ou Técnica de Takeuchi, que consiste na criação de um túnel dentro da artéria pulmonar, de forma a conectar a artéria

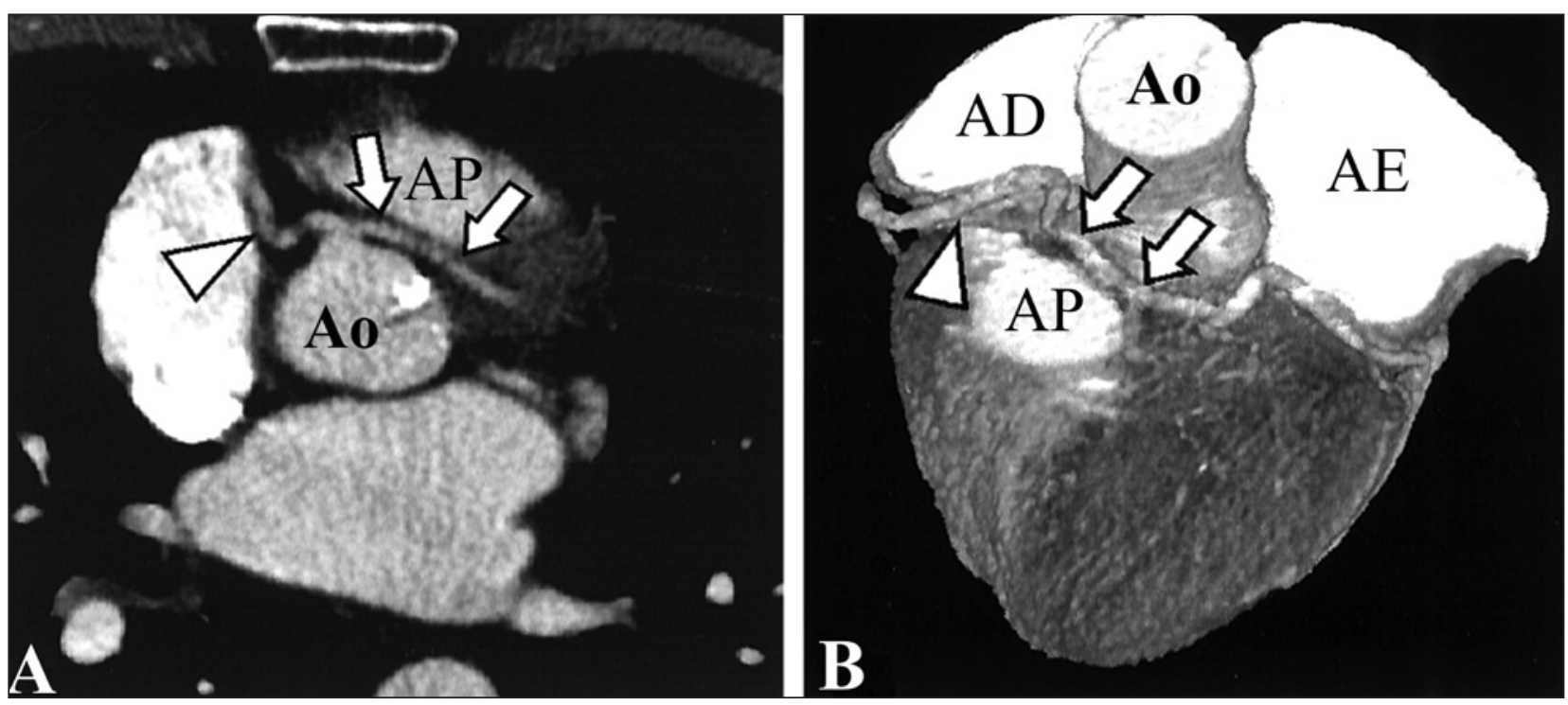

Figura 2 - Origem anômala da artéria coronária esquerda com curso interarterial visualizada por TCAC. A: corte axial para avaliação da origem das artérias coronárias. B: reconstrução tridimensional. As setas indicam o trajeto da artéria descendente anterior entre a aorta ascendente e o tronco da artéria pulmonar. A cabeça da seta indica a artéria coronária esquerda. Ao: aorta; AP: artéria pulmonar; AD: átrio direito; AE: átrio esquerdo(adaptado de Ropers et al. ${ }^{24}$ ). 
coronária esquerda com a aorta ${ }^{11,13,37-40}$. Outras opções cirúrgicas são a simples ligadura da artéria coronária esquerda e o implante de enxertos arteriais e veno$\operatorname{sos}^{11,37-40}$. A mortalidade cirúrgica para todas as técnicas combinadas variava de 75 a $80 \%$, na década de $80^{37}$. Atualmente, ela situa-se entre 0 e $23 \%{ }^{37}$.

A evolução clínica e a recuperação funcional do ventrículo esquerdo dependem do grau de disfunção ventricular pré-operatória. Na maioria dos casos, há uma normalização da função ventricular no pós-operatório ${ }^{11,13,37-40}$.
Os portadores de OACEA, em geral, são assintomáticos ${ }^{12}$. Mesmo em atletas, apenas a minoria é sintomática ${ }^{12}$. E, nos indivíduos sintomáticos, o ECG e os exames indutores de isquemia geralmente são normais $^{8,12,28}$. A presença de sintomas sugestivos da doença, sobretudo em atletas, deve ser investigada com exames de imagem. Na impossibilidade do ecocardiograma diagnosticar a origem anômala, deve-se realizar TCAC, RNM ou CA de artérias coronárias ${ }^{8,28}$.

Não há consenso na literatura para o manejo dos

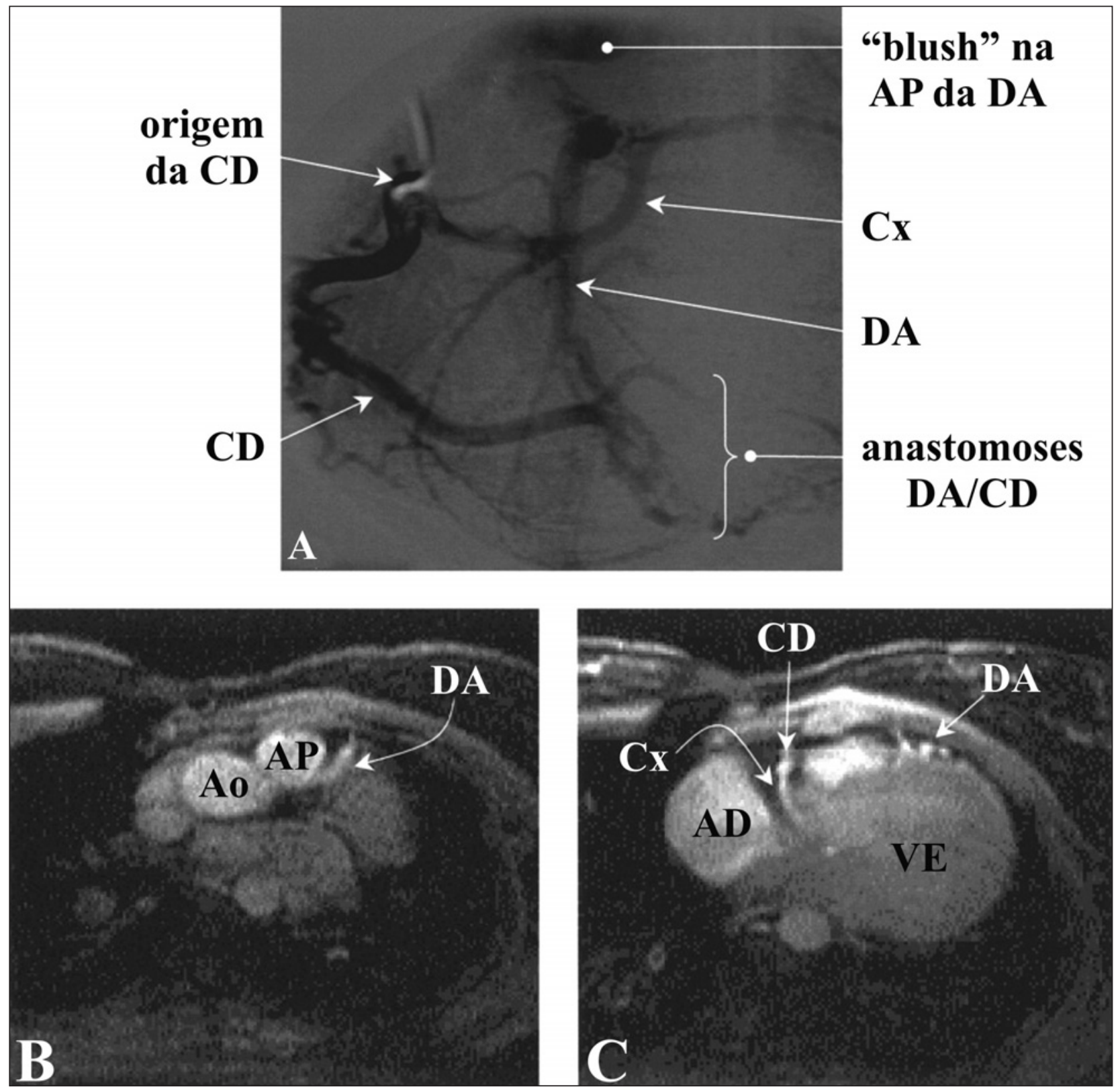

Figura 3 - Atresia do TCE em um paciente portador de valva aórtica bicúspide e ducto arterioso patente. A: cineangiocoronariografia mostrando que a artéria circunflexa(Cx) origina-se da artéria coronária direita(CD). A artéria descendente anterior(DA) enche-se retrogradamente e forma uma fístula com a artéria pulmonar(AP). B: no corte axial da RNM, observa-se uma falsa inserção da DA na AP. C: imagem axial da Cx originando-se da CD. Ao: aorta; AD: átrio direito; VE: ventrículo esquerdo(adaptado de Taylor et al. ${ }^{17}$ ) 
portadores de OACEA assintomáticos. Nos indivíduos sintomáticos, deve-se optar pelo tratamento cirúrgi$\mathrm{CO}^{12,28}$. As opções cirúrgicas são o implante de enxerto arterial ou venoso, o reimplante da artéria coronária anômala no seio coronariano anatômico, plastia do segmento intramural da artéria coronária e translocação do tronco da artéria pulmonar ${ }^{12,28}$. Não há dados que definam a melhor técnica cirúrgica.

\section{CONCLUSÃO}

A origem anômala das artérias coronárias é uma doença rara, mas potencialmente letal se não diagnosticada e tratada precocemente. As crianças e os atletas jovens constituem um grupo de risco para as seqüelas catastróficas. Nestes pacientes, a terapêutica cirúrgica traz bons resultados, sendo o tratamento definitivo. A identificação da doença em pacientes assintomáticos permanece um desafio. E novos estudos são necessários para definição do tratamento ideal nestes pacientes.

\section{REFERÊNCIAS BIBLIOGRÁFICAS}

1. Dawn B, Talley JD, Prince CR, Hoque A, Morris GT, Xenopoulos NP, et al. Two-dimensional and doppler transesophageal echocardiographic delineation and flow characterization of anomalous coronary arteries in adults. J Am Soc Echocardiogr. 2003;16(12):1274-86.

2. Hayashi JH, Abreu Fo LM, Sumita MK, Takimura CK, Hotta $\mathrm{VT}$, Forte AAC. Incidência de anomalias coronárias em hospital geral. Rev Bras Cardiol Invas. 2002;10 (3):26-32.

3. Yamanaka O, Hobbs RE. Coronary artery anomalies in 126.595 patients undergoing coronary arteriography. Cathet Cardiovasc Diagn. 1990;21(1):28-40.

4. Baltaxe HA, Wixson D. The incidence of congenital anomalies of the coronary arteries in the adult population. Radiology. $1977 ; 122(1): 47-52$.

5. Leberthson RR, Dinsmore RE, Bharati S, Rubenstein J, Caulfield J, Wheeler BO, et al. Aberrant coronary artery origin from the aorta. Diagnosis and clinical significance. Circulation. 1974;50(4):774-9.

6. Engel HJ, Torres C, Page HL Jr. Major variations in anatomical origin of the coronary arteries: angiographic observations in 4.250 patients without associated congenital heart disease. Cathet Cardiovasc Diagn. 1975;1(2):157-69.

7. Chaitman BR, Lesperance J, Saltiel J, Bourassa MG. Clinical, angiographic and hemodynamic findings in patients with anomalous origin of the coronary arteries. Circulation. 1976;53 (1):122-31.

8. Angelini P, Velasco JA, Flamm S. Coronary anomalies: incidence, pathophysiology, and clinical relevance. Circulation. 2002;105(20):2449-54.

9. Maron BJ. Sudden death in young athletes. N Engl J Med. 2003;349(11):1064-75.

10. Oliveira SA, Snitcowsky R. Origem anômala da artéria coronária esquerda do tronco pulmonar. In: Macruz R, Snitcowsky R, eds. Cardiologia pediátrica. 1aㅡ ed. São Paulo:Sarvier;1983. p.508-43.

11. Takimura $\mathrm{CH}$, Nakamoto A, Hotta VT, Campos MF, Málamo M, Otsubo R. Origem anômala da artéria coronária esquerda no tronco pulmonar. relato de um caso em adulto. Arq Bras Cardiol. 2002;78(3):309-11.

12. Erez E, Tam VK, Doublin NA, Stakes J. Anomalous coronary artery with aortic origin and course between the great arteries: improved diagnosis, anatomic findings, and surgical treatment. Ann Thorac Surg. 2006;82(3):973-7.

13. Amaral F, Carvalho JS, Granzotti JA, Shinebourne EA. Origem anômala da artéria coronária esquerda do tronco pulmonar: perfil clínico e resultados a médio prazo do tratamento cirúrgico. Arq Bras Cardiol. 1999;72(3):307-20.

14. Ogden JA. Congenital anomalies of the coronary arteries. Am J Cardiol. 1970;25(4):474-9.

15. Dodge-Khatami A, Mavroudis C, Backer CL. Congenital Heart Surgery Nomenclature and Database Project: anomalies of the coronary arteries. Ann Thorac Surg. 2000;69(4 Suppl): S270-97.

16. Shi H, Aschoff AJ, Brambs HJ, Hoffmann MH. Multislice CT imaging of anomalous coronary arteries. Eur Radiol. 2004;14 (12):2172-81.

17. Taylor AM, Thorne SA, Rubens MB, Jhooti P, Keegan J, Gatehouse PD, et al. Coronary artery imaging in grown up congenital heart disease: complementary role of magnetic resonance and X-ray coronary angiography. Circulation. 2000;101(14):1670-8.

18. Kruskal JB, Hartnell GG. Nonatherosclerotic coronary artery disease: more than just stenosis. Radiographics. 1995;15(2): 383-96.

19. Ropers D, Moshage W, Daniel WG, Jessl J, Gottwik M, Achenbach S. Visualization of coronary artery anomalies and their anatomic course by contrast-enhanced electron beam tomography and three-dimensional reconstruction. Am J Cardiol. 2001;87(2):193-7.

20. Dabizzi RP, Teodori G, Barletta GA, Caprioli G, Baldrighi G, Baldrighi V. Associated coronary and cardiac anomalies in the tetralogy of Fallot. An angiographic study. Eur Heart J. 1990;11(8):692-704.

21. Carvalho JS, Silva CM, Rigby ML, Shinebourne EA. Angiographic diagnosis of anomalous coronary artery in the tetralogy of Fallot. Br Heart J. 1993;70(1):75-8.

22. Ruiz CE, Lau FYK. Congenital atresia of left main coronary artery: proposed mechanism for severe disabling angina in a patient with non-atherosclerotic single right coronary artery - a case report. Cathet Cardiovasc Diagn. 1991;23(3):190-3.

23. Ghosh PK, Friedman M, Vidne BA. Isolated congenital atresia of the left main coronary artery and atherosclerosis. Ann Thorac Surg. 1993;55(6):1564-5.

24. Ropers D, Gehling G, Pohle K, Maeffert R, Regenfus M, Moshage $W$, et al. Anomalous course of the left main or left anterior descending coronary artery originating from the right sinus of Valsalva: identification of four common variations by electron beam tomography. Circulation. 2002;105(6): e42-3.

25. Memisoglu E, Ropers D, Hobikoglu G, Tepe MS, Labovitz AJ. Usefulness of electron beam computed tomography for diagnosis of an anomalous origin of a coronary artery from the opposite sinus. Am J Cardiol. 2005;96(10):1452-5.

26. Kasprzak JD, Kratochwil D, Peruga JZ, Drozdz J, Rafalska K, Religa W, et al. Coronary anomalies diagnosed with transesophageal echocardiography: complementary clinical value in adults. Int J Card Imaging. 1998;14(2):89-95.

27. Popma JJ, Bittl J. Coronary angiography and intravascular ultrasonography. In: Braunwald E, Zipes DP, Libby P, eds. Heart disease. $6^{\text {th }}$ ed. Philadelphia: W.B. Saunders;2001. p.387-421.

28. Basso C, Maron BJ, Corrado D, Thiene G. Clinical profile of congenital coronary artery anomalies with origin from the wrong aortic sinus leading to sudden death in young competitive athletes. J Am Coll Cardiol. 2000;35(6):1493-501. 
29. Johnsrude CL, Perry JC, Cecchin F, Smith EO, Fraley K, Friedman RA, et al. Differentiating anomalous left main coronary artery originating from the pulmonary artery in infants from myocarditis and dilated cardiomyopathy by electrocardiogram. Am J Cardiol. 1995;75(1):71-4.

30. Berbarie RF, Dockery WD, Johnson KB, Rosenthal RL, Stoler RC, Schussler JM. Use of multislice computed tomographic coronary angiography for the diagnosis of anomalous coronary arteries. Am J Cardiol. 2006;98(3):402-6.

31. Post JC, van Rossum AC, Bronzwaer JG, de Cock CC, Hofman $\mathrm{MB}$, Valk J, et al. Magnetic resonance angiography of anomalous coronary arteries: a new gold standard for delineating the proximal course? Circulation. 1995;92(11):3163-71.

32. McConnell MV, Ganz P, Selwyn AP, Li W, Edelman RR, Manning WJ. Identification of anomalous coronary arteries and their anatomic course by magnetic resonance coronary angiography. Circulation. 1995;92(11):3158-62.

33. Vliegen HW, Doornbos J, Ross A, Jukema JW, Bekedam MA, van der Wall EE. Value of fast gradient echo magnetic resonance angiography as an adjunct to coronary arteriography in detecting and confirming the course of clinically significant coronary artery anomalies. Am J Cardiol. 1997;79(6):773-6.

34. Bunce $\mathrm{NH}$, Lorenz $\mathrm{CH}$, Keegan J, Lesser J, Reyes EM, Firmin $\mathrm{DN}$, et al. Coronary artery anomalies: assessment with free- breathing three-dimensional coronary MR angiography. Radiology. 2003;227(1):201-8.

35. Pinto IMF, Sousa AGMR, Ishikawa W, Sasdelli Neto R, Piva e Mattos LA, Sousa JEMR. Ressonância magnética e tomografia computadorizada no diagnóstico de insuficiência coronária. Rev Bras Cardiol Invas. 2006;14(2):168-77.

36. Hamon M, Biondi-Zoccai GGL, Malagutti P, Agostini P, Morello $R$, Valgimigli $M$, et al. Diagnostic performance of multislice spiral computed tomography of coronary arteries as compared with conventional invasive coronary angiography: a metaanalysis. J Am Coll Cardiol. 2006;48(9):1896-910.

37. Dodge-Khatami A, Mavroudis C, Backer CL. Anomalous origin of the left coronary artery from the pulmonary artery: collective review of surgical therapy. Ann Thorac Surg. 2002;74(3):946-55.

38. Moraes F, Lincoln C. Anomalous origin of left coronary artery: evolution of surgical treatment. Eur J Cardiothorac Surg. 1996;10(8):603-8.

39. Reul RM, Cooley DA, Hallman GL, Reul GJ. Surgical treatment of coronary artery anomalies: report of $37 \quad 1 / 2$-year experience at the Texas Heart Institute. Tex Heart Inst J. 2002;29(4):299-307.

40. Selzman $\mathrm{CH}$, Zimmerman MA, Campbell DN. ALCAPA in an adult with preserved left ventricular function. J Card Surg. 2003;18(1):25-8. 